\title{
Efficient Cloud Data Storage in Clustering Analysis Method
}

\author{
Kiruthiga.G \\ Assistant Professor \& Research Scholar, \\ Department of Computer Applications, \\ Guru Nanak College, Chennai. \\ Email: g_kiruthiga@yahoo.co.in
}

\author{
Dr. Mary Vennila.S \\ Associate Professor \& Research Supervisor, \\ PG \& Research Department of Computer Science, \\ Presidency College, Chennai. \\ Email: vennilarhymend@yahoo.co.in
}

\begin{abstract}
Cloud information garage is service where facts is remotely maintained, managed, and backed up. The cloud provider permits the users to preserve documents on line, that allows accessing them from any vicinity through the net. Distributed computing and numerous customers expect that distributed computing will reshape information innovation techniques. huge amount of records is put away inside the cloud which wants to be recovered effectively. The recovery of data from cloud takes a considerable measure of time as the information isn't put away in a sorted out way. Information mining is in this manner critical in cloud computing. We can join data mining and distributed computing (included records Mining and Cloud Computing- IDMCC) with a view to offer agility and brief access to the era. With the cloud computing generation, customers use a diffusion of gadgets, which includes desktops, laptops, clever phones, and PDAs to get entry to programs, garage, and application-improvement systems over the net, through services presented via cloud computing carriers. Blessings of the cloud computing generation include price savings, high availability, and clean scalability.Thus in this presented work a survey is introduced for cloud data storage, and their cluster analysis for utilizing the data into various business intelligence applications. This paper suggests a new model of cluster analysis of data is proposed which provides the clustering as service.
\end{abstract}

Key Words: cloud computing, cloud storage, clustering, types of clustering

\section{INTRODUCTION}

Large volume of information is put away in the cloud condition and should be recovered effectively. The recovery of data from cloud takes a considerable measure of time as the information is not put away in a sorted out way. Data Clustering is a technique of analysing facts and extraction of significant patterns from the raw units of facts. The significant is named right here to suggest the styles or expertise recovered from the training samples that is further used to become aware of the similar pattern which belongs to the discovered pattern. In the information clustering two predominant forms of learning techniques are discovered namely supervised gaining knowledge of method and unsupervised mastering method. These learning models are used to evaluate data and create a mathematical model for utilizing to identify the similar data patterns arrived for classifying them in some pre-fined groups.

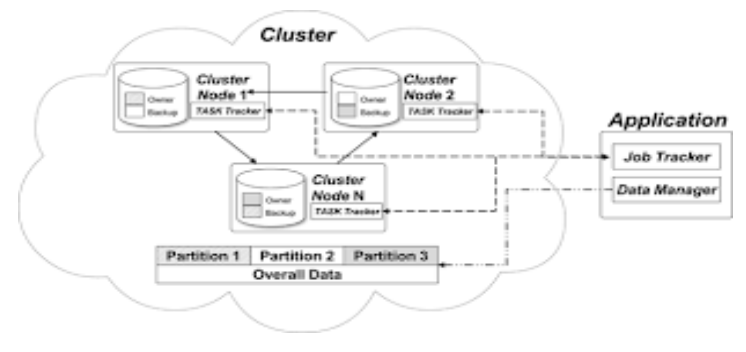

Cluster partition 
In supervised learning technique the data is processed with their class labels and here the class labels are working as teacher for learning algorithm. On the other hand in unsupervised learning technique the data not contains the class labels to utilize as the teacher. Therefore using the similarity and dissimilarity of the input training samples the data is categorized. Therefore the supervised learning processes are known as the classification of data and the unsupervised learning techniques are supporting the cluster analysis of data. In this presented work the unlabelled data is used for analysis therefore the data analysis technique is used as the cluster analysis. Clustering is the unsupervised classification of patterns or input samples. That can used classify observations, data items, or feature vectors into groups. These groups are in data mining is known as the cluster analysis of data. Within the case of clustering, the problem is to organization a given series of unlabelled patterns into significant clusters. In a feel, labels are associated with clusters additionally, but these class labels are data pushed; that is, they're obtained entirely from the statistics.

\subsection{Clustering technique background}

Clustering is a most famous facts mining technique used to discover beneficial unknown pattern from records in big repository. Clustering is grouping of facts into special clusters such that elements belong to equal cluster are most comparable at the same time as elements belongs to extraordinary cluster are varied. Basically Clustering strategies are divided into two large categories. si) Hard clustering ii) Soft Clustering. In difficult Clustering, each report can belong to simplest one Cluster. Hard Clustering is likewise referred to as exclusive clustering. In tender Clustering equal file can belong to more than one group. it is also known as Overlapping Cluster technique.

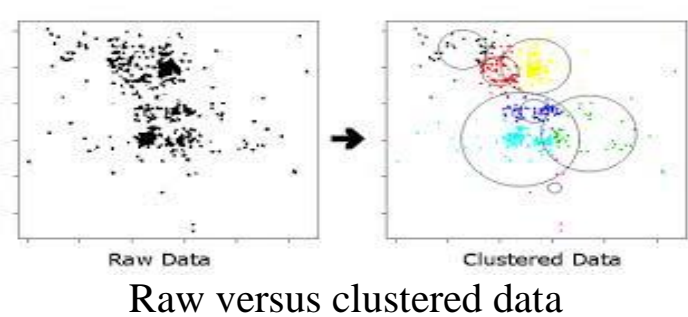

This section provides the overview of the introduction of data clustering and the selected domain for study in data storage. In the next section the different kinds of clustering algorithms are learned for understanding the technique behind the cluster analysis.

\subsection{Types of clustering technique}

There are a significant amount of clustering algorithms and methods are available some essential techniques are described:

\subsubsection{Partitioning Method}

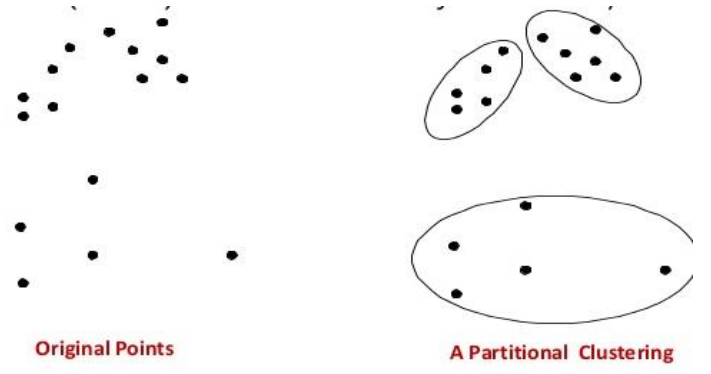

In this clustering approach the $\mathrm{n}$ numbers of data or objects are provided, and $\mathrm{k}$ number of partitions are required from the data but the number of partition is such that $\mathrm{k} \leq \mathrm{n}$. This means the partitioning algorithm will generate $\mathrm{k}$ partitions satisfying below condition: a. Each group have minimum one object. b. Each object should be a member of exactly one group.

\subsubsection{Hierarchical Methods}

Hierarchical method
hierarchically manner of $\begin{array}{r}\text { generates } \\ \text { clusters }\end{array}$ organization. That can be achieved using the following manner: 
a. Agglomerative Approach

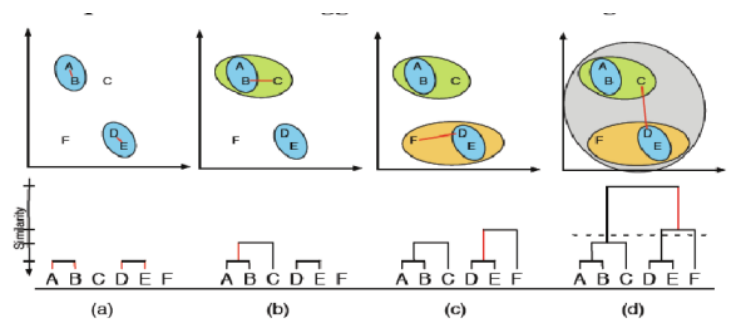

It follows the bottom-up approach. Firstly, it generates separate group for each object of data. Next, it merges these groups on the basis of closer similarities. This process is repeated till the entire crowd of groups are not combined in a single or until the termination condition holds.

\section{b. Divisive Approach}

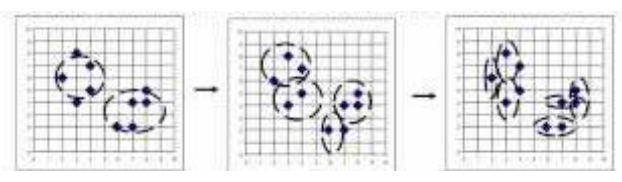

It follows the topdown approach. Process starts with a single cluster having all data objects. Then, it continues splitting the bigger clusters into smaller ones. This technique maintains until the termination situation holds. This technique is inflexible that is after merge or split is finished, it is able to never be negated.

\subsubsection{Density-Based Methods}

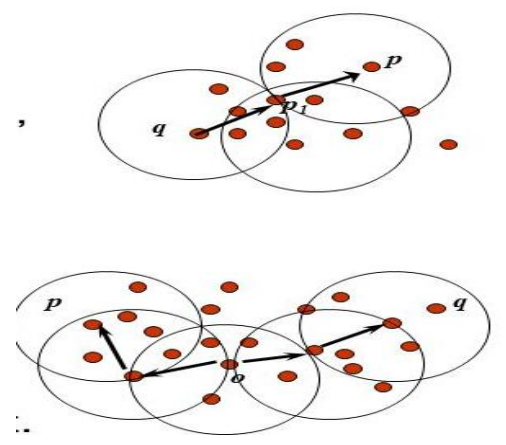

This technique uses the perception of density. The main design is to keep expanding the cluster until the density of neighbourhood reaches certain threshold i.e. within a given cluster, the radial span of a cluster must possess certain number of points for each data points.

\subsubsection{Grid-Based Method}

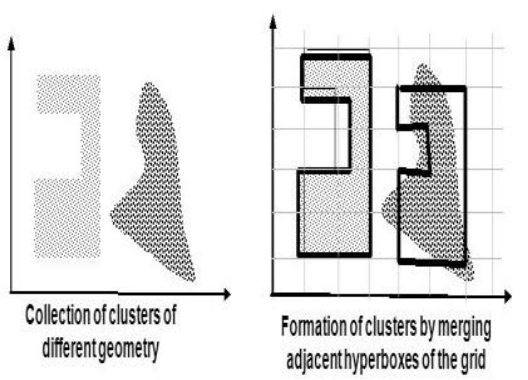

This approach quantizes the object area into a big no. of cells which collectively nurture a grid. The method having the flowing advantages:

- This approach provides is its speedy processing.

- The handiest dependability is depending upon the no. of cells in item space.

\section{Model-Based Methods}

In version-based scheme, a version may be conjectured for each cluster together with that; it then identifies statistics fitting great into that model.

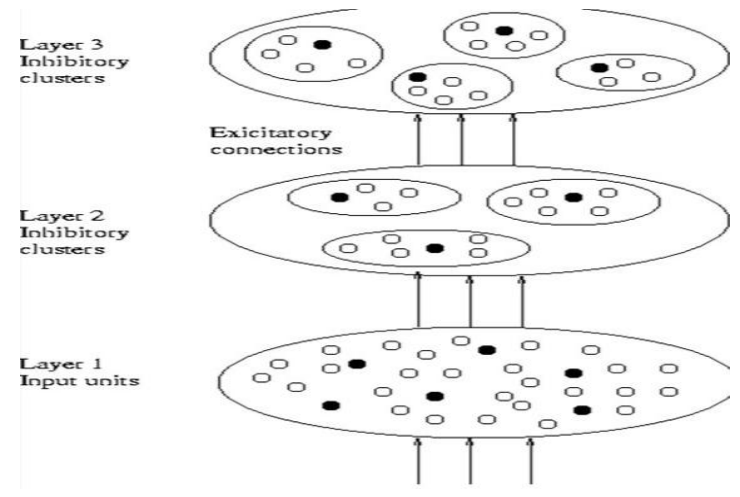

This approach component a means to routinely display quantity of clusters derived from the standard data, considering outlier or noise. As a end result, it creates strong clustering techniques. 


\subsubsection{Constraint-Based Method}

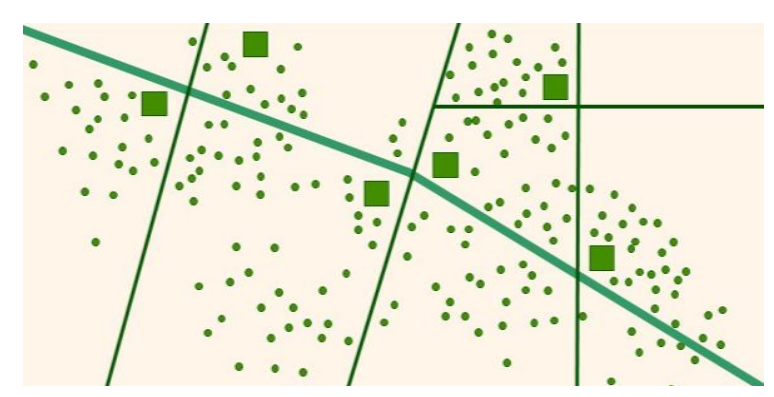

It performs clustering on the basis of constraints either application oriented or user oriented. These constraints are actually the prospect or properties of the desired clustering results. These constraints make communication with the clustering process easy.

\section{LITERATURE SURVEY}

One of the cloud services that are being offered is a storage method for the data. Earlier to the idea of cloud computing critical industrial data was stored internally on the garage media [1]. From music documents to pics to sensitive files, the cloud invisibly backs up all the documents and folders and eliminates the need for infinite and costly look for more garage area. Whilst there are widespread facts, garage cloud alleviates shopping for an external tough drive or deleting old files to make room for the new ones. Thus many organizations have entered in the cloud environment for the storage service. These organizations pay for the amount of space they use in the cloud. Distributed storage is helpful and financially savvy. It works by putting away the records on a server some place in the web instead of on the nearby hard drive. This permits moving down, match up, and getting to information over different gadgets as long as clients have web ability.
In cloud computing various researches have been made to improve the performance of cloud computing. Various statistics mining algorithms had been implemented in various ways to manipulate the large amount of facts in cloud. The related works on this discipline are: Bhupendrapanchal and R.ok Kapoor [2] proposed clustering and caching methodologies for enhancing the performance. the main concept is to make replicas of statistics to be had at each information centres, so even though one information middle goes down, everything within the second statistics center is clustered with the primary Kashish Ara Shakil and MansafAlam [3] proposed an approach that gives management of cloud facts via clustering and uses a k-median as clustering method. A.Mahendiran et al [4] proposed the implementation of ok-manner clustering algorithm in cloud computing for huge datasets. Kriti Srivastava [5] proposed the implementation of agglomerative hierarchical clustering set of rules to enable the advantages which include scalability, elasticity and managing large datasets.

\section{PROPOSED MODEL IMPROVING SUPERVISED LEARNING ALGORITHMS WITH CLUSTERING}

Clustering is an unsupervised machine learning approach, however would it be able to be utilized to enhance the exactness of regulated machine learning calculations too by grouping the information focuses into comparable gatherings and utilizing these bunch names as free factors in the managed machine learning calculation. Allow's test out the impact of bunching on the exactness of our adaptation for the arrangement inconvenience utilizing 3000 perceptions with 100 indicators of stock actualities to foreseeing regardless of whether the stock will leave behind or 
down the use of $R$. This dataset incorporates a hundred unprejudiced factors from X1 to X100 speaking to profile of a stock and one last outcomes variable $\mathrm{Y}$ with levels: 1 for upward push in stock rate and - 1 for drop in stock rate.

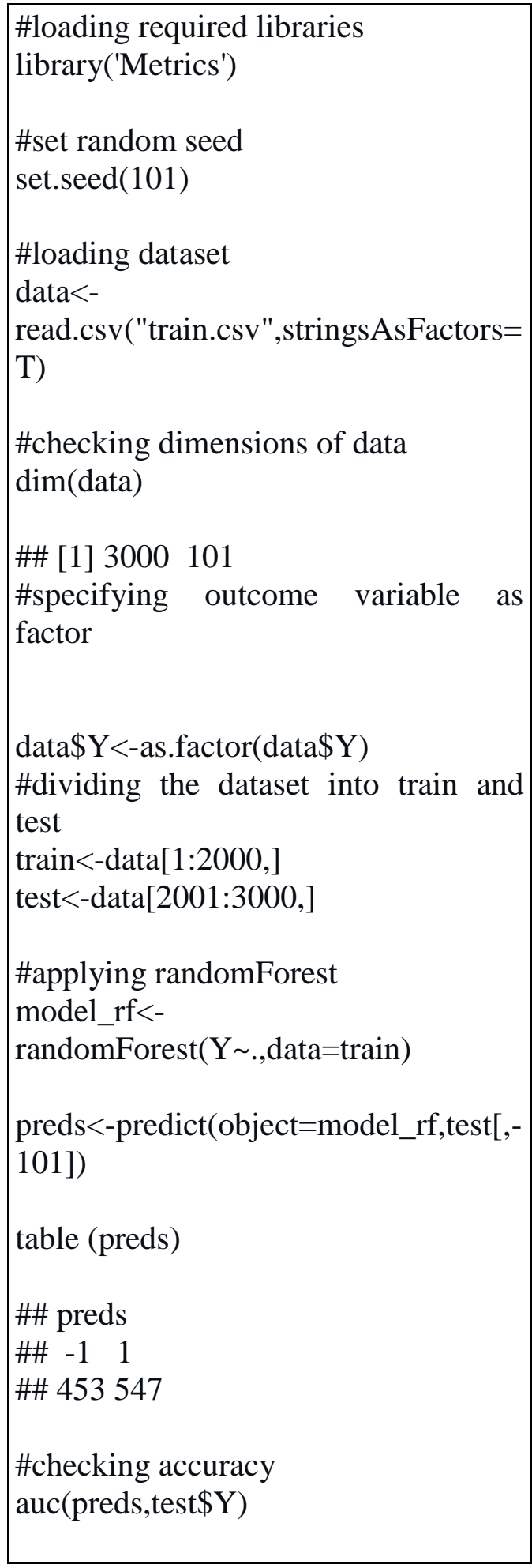

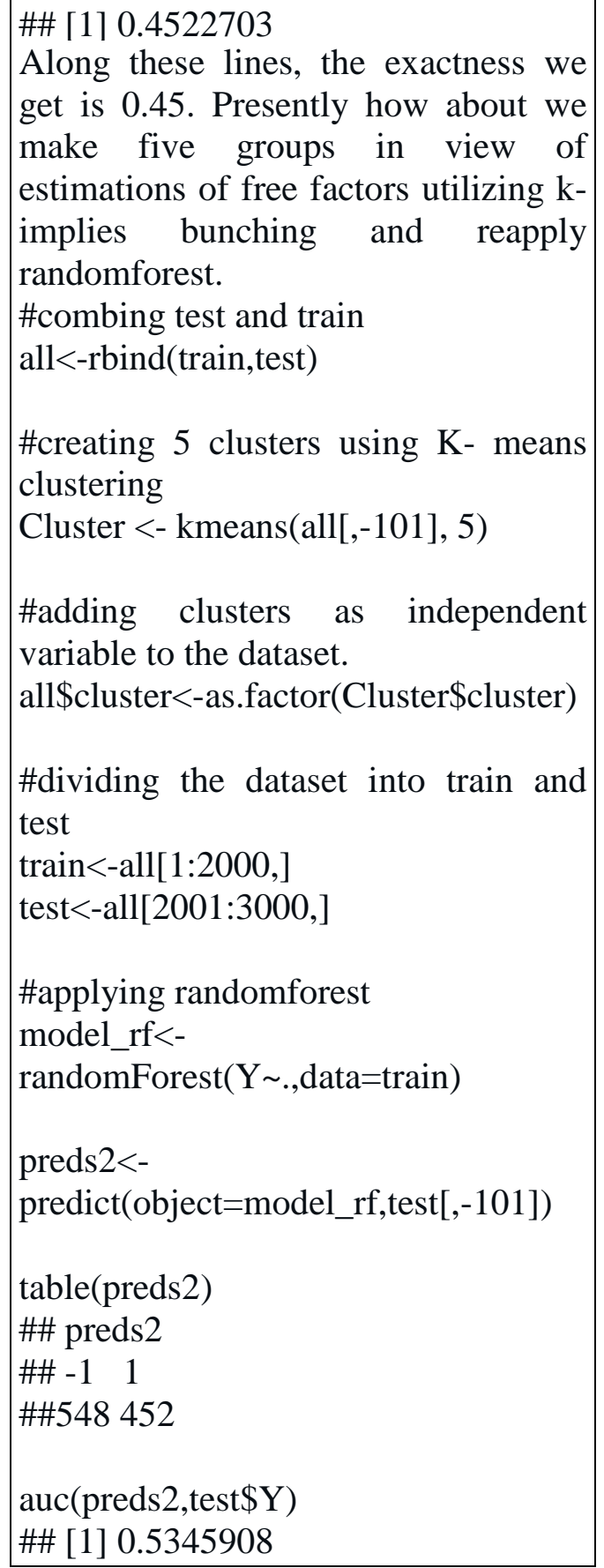

Despite the fact that the last precision is poor however bunching has given our model a huge lift from exactness of 0.45 to somewhat over 0.53 . This demonstrates bunching can without a doubt be useful for regulated machine learning undertakings.

We have examined what the different methods for performing bunching are. It discovers applications for unsupervised 
learning in a vast no. of areas. You likewise perceived how you can enhance the precision of your directed machine learning calculation utilizing grouping.

\section{CONCLUSION AND FUTURE WORK}

Despite the fact that clustering is straightforward to put into effect, the need to attend a few crucial factors like treating exceptions for your data and guaranteeing each group has adequate people. The proposed approach has advantages find it irresistible presents rapid access to information, presents the information of utilization of cloud garage area, scalability and helps in mining huge statistics units which are heterogeneous in nature. Future works for the proposed model is to apply other clustering algorithms within the cloud storage and evaluate the outcomes to discover the excellent clustering algorithm for cloud garage.

\section{REFERENCES}

[1] NaskarAnkita, Mrs. Mishra Monika R, "using cloud computing to provide data mining services" published in international journal of engineering and computer science, volume 2 issue 3 march 2013

[2] Bhupendra Panchal, R.K Kapoor, "Performance Enhancement of cloud computing with clustering" published in international journal of engineering and advanced technology, volume-2, issue-5, June 2013

[3] Kashish Ara Shakil, ManasafAlam, "data management in cloud based environment using $\mathrm{k}$ median clustering technique" published in "international journal of computer
Applications 4th International IT Summit Confluence 2013- The Next Generation Information Technology Summit"

[4] A.Mahendiran, N.Saravanan, N.Venkata Subramanian and Sairam, "Implementation of K-means Clustering in cloud computing environment" published in research journal of applied sciences, engineering and technology

[5] Kriti Srivastava, R. Shah, D. Valia, and H. Swaminarayan," Data Mining Using Hierarchical Agglomerative Clustering Algorithm in Distributed Cloud Computing Environment" published in," International Journal of Computer Theory and Engineering, Vol. 5, No. 3, June 2013"

[6] Amazon S3.http://aws.amazon.com/s3/

[7] P. Bo, C. Bin and L. Xiaoming, "Implementation Issues of A Cloud Computing platform", In Bulletin of the IEEE Computer Society Technical Committee on Data Engineering, 2009.

[8] Marina Meil ‘a, "The stability of a good clustering", Journal of Artificial Intelligence Research (1993) Submitted 6/91; published 9/91

[9] [1] Ruxandra Stefania PETRE, "Data Mining in Cloud Computing" published in "Database Systems Journal vol.III, no.3/2012"

[10]Shynu, P. G., and K. John Singh.2016 "A Comprehensive Survey and Analysis on Access Control Schemes in Cloud Environment." Cybernetics and Information Technologies 16(1), pp.19 38. 